\title{
The impact of tinnitus on daily activities in adult tinnitus sufferers: A pilot study
}

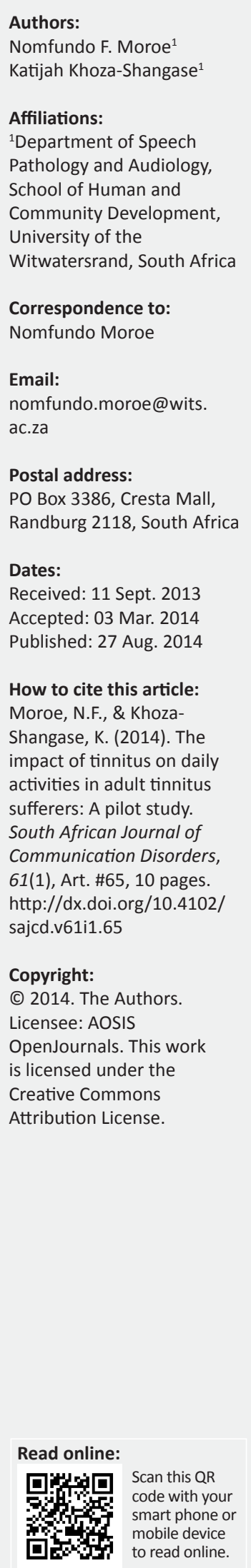

Background: Few South African studies have been published on the impact of tinnitus on quality of life of tinnitus sufferers, although evidence suggests that a large portion of the general population suffers from tinnitus.

Objectives: The current study aimed at describing the effects of tinnitus on the quality of life of the participants as measured by the Tinnitus Handicap Inventory (THI).

Method: In a cross-sectional descriptive study design, 27 participants took part in the study by completing a self-administered THI questionnaire and participating in a semi-structured interview. Descriptive and inferential statistics were used to analyse the data. Descriptively, content analysis was used to organise and convey results from the interviews.

Results: Participants reported a wide range of perceived disability on the THI. Results ranged from mild to catastrophic, with functional disability being most prominent in all participants, although there were differences when results were analysed according to gender. There was an association between gender and the type of perceived disability, although this was statistically non-significant $(p>0.05)$. Only $26 \%$ of the participants reported no effect on occupational performance and quality of life, with the remainder of the participants reporting a significant effect. Limited effective management strategies were reported to have been implemented - a significant implication for the audiologists.

Conclusion: The results have implications for audiologists as they suggest that audiologists should take a detailed case history to determine the extent to which tinnitus affects the individual. Furthermore, audiologists should administer a scale such as the THI in the management of tinnitus.

\section{Introduction}

Tinnitus is an auditory perception of continuous or intermittent sound in the absence of any external acoustic stimulation (Baldo, Doree, Lazzarini, Molin \& McFerran, 2006), with at least one-third of the general population reported to have experienced tinnitus once in their life time (Martines, Bentivegna, Martines, Sciacca \& Martinciglio, 2010). Its prevalence is reported to increase with age and is said to be much higher than the number of patients who seek treatment (Krog, Engdahl \& Tambs, 2010). Although its aetiology is still not fully understood, it is well documented that tinnitus and hearing loss are often associated (Martines et al., 2010). It can be argued that it is probably because of its poorly understood aetiology that no single treatment method has proved to be an overall solution to the problem (Stevinson, 2003); and it possibly is the reason why a number of uncured tinnitus sufferers still frequent the ear, nose and throat (ENT) specialists' clinics.

Despite the fact that the aetiology of tinnitus is not yet fully understood, there is growing evidence that, regardless of the cause, tinnitus does negatively impinge on a sufferer's quality of life. Various researchers have argued and demonstrated that tinnitus is a distressing symptom which may significantly affect the quality of life of the sufferer (Martines et al., 2010; Pinto, Sanchez \& Tomita, 2010). In some cases, individuals who experience severe or chronic tinnitus may perceive themselves as handicapped (Roggerone, 2010). This negative effect that tinnitus has on the sufferer could partly be due to the fact that tinnitus is viewed as a chronic multifactorial condition which can be induced by all types of hearing loss as well as by somatic and psychiatric disorders and pharmaceutical drugs.

The impact of tinnitus on quality of life has been documented in patients who present with decompensated (chronic) tinnitus; and not in those suffering from compensated (acute) tinnitus (Stobik, Weber, Münte, Walter \& Frommer, 2005). With compensated tinnitus the patient does not seem to be bothered by the sound and grows accustomed to it easily (Gopinath, McMahon, Rochtchina, Karpa \& Mitchell, 2010), whereas in the case of decompensated tinnitus, the 
most severe form of tinnitus, patients report that it causes debilitating problems (Gopinath et al., 2010; Hiller \& Goebel, 2007; Westin, Hayes \& Andersson, 2008). These debilitating problems include depression, anxiety, frustration, insomnia; and may also result in suicide or reduced cognitive function. These problems in turn may affect the quality of life of the affected individuals.

A number of studies have established the relationship between tinnitus and quality of life. Psychological scales have found a correlation between the severity of tinnitus and a number of health problems (Erlandsson \& Holgers, 2001). Amongst these health problems are distress symptoms such as headaches, dizziness and insomnia (Dineen, Doyle \& Bench, 1997; Erlandsson, Hallberg \& Axelsson, 1992). In a study of a cohort of older adults with tinnitus, a significant association between tinnitus and the quality of life was established (Nondahl et al., 2007). However, in another study by Gopinath et al. (2010), the findings highlighted the view that the effects of tinnitus on the quality of life are highly individualised, and that the individual's personality characteristics are more likely to predispose some individuals to experience tinnitus as a distressing symptom than in others. Regardless of this individualisation of experiences, Gophinath et al. (2010), argue that tinnitus may cause depression in sufferers as its presence may have a negative impact on the patient's desire to interact with other people, making social interaction more difficult. This may in turn affect daily activities such as occupational performance, driving, eating and performing general tasks. Jacobson and McCaslin (2001) emphasise the gravity of the symptom by asserting that it is not uncommon to read in the literature anecdotal reports of patients with tinnitus of a severity that caused them to consider, attempt or proceed to commit suicide. These authors argue, however, that it is unlikely that tinnitus alone could cause distress of a magnitude to cause a patient to commit suicide. They contend that in such cases it is more likely that comorbid conditions such as clinical depression would serve to increase a patient's reaction to tinnitus. Jastreboff and Hazell (1993) argue that emotional factors are likely to influence the manner in which the brain processes the information from the ear, because the auditory system has a number of connections with the limbic system, which is involved in emotions.

A retrospective study by Lewis, Stephens and Mckenna (1994) reported that 28 tinnitus sufferers had committed suicide. These authors suggest that 'depressed people with tinnitus are more likely to come to the attention of doctors, as they are less resilient and less able to cope with chronic discomfort' (Lewis et al., 1994, p. 35). Newman et al. (2011) recommend that tinnitus cannot be overlooked when assessing patients with hearing loss. These authors stress that the clinician should be prepared to address suicidal ideas, plans, means, intentions and safety measures with patients, whereas Jacobson and McCaslin (2001) suggest that the practising audiologists need to be aware of the possible risk of suicide amongst clinically depressed tinnitus sufferers. Hence the importance of using tinnitus assessment scales that explore patients' perception of the impact of their tinnitus on their lives.
Meikle (2003) and Ramkumar and Rangasayee (2010) argue that standardising assessment and classification would provide a rational basis for stratifying tinnitus patients into different groups and would further enable meta-analysis and other types of comparison between treatment centres. McDougall, Horgan, Baldwin, Tucker and Frid (2008) recommend using the new International Classification of Functioning, Disability and Health (ICF) model for this purpose. This interactive model sees the person's disability as a dynamic interaction between health conditions and personal factors. According to this model, disability is an umbrella term covering three levels of reduced function:

- impairment of the body structure or function (body level)

- limitations in activities (person level)

- restriction of participation (society level) (Ramkumar \& Rangasayee, 2010).

The impact of tinnitus on function can be conceptualised for a typical patient using the ICF (Ramkumar \& Rangasayee, 2010). When one adopts this interactive model, tinnitus is the 'health condition' and the 'problem in body function' for the patient is the perception of tinnitus as a loud ringing sound. The patient's main tinnitus-related problem is that the tinnitus makes it hard to concentrate (activity limitation), and in turn the concentration difficulty hinders work performance (participation restriction). The main environmental factor influencing the patient's tinnitus is that the family is unsympathetic toward the tinnitus condition and does not provide emotional support (Henry, Dennis \& Schechter, 2005), possibly because it is an invisible condition, unless the patient suffers from objective tinnitus which can be heard not only by the sufferer but also by an independent person. According to the ICF interactive model, 100\% of individuals with chronic tinnitus have impaired body function because of the condition. For the $20 \%$ of individuals with clinically significant tinnitus, the associated problems have an impact to some degree at the activity-limitation level (Davies \& Refaie, 2000). It is for these reasons that the current study was conceptualised. Its aim was to describe the perceived effects of tinnitus on daily activities, such as occupational performance in adults suffering from tinnitus, as measures by the Tinnitus Handicap Inventory (THI).

Quinn and Ryan (2003) argue that the evaluation of a patient with tinnitus should start with a carefully taken history. The patient's description of the tinnitus is important, as it can provide key information during the initial evaluation. These authors argue that the examiner should enquire about the quality of the sound, whether the tinnitus is pulsatile or non-pulsatile, the perceived location, the pitch, the loudness, onset, episodic or constant, alleviating or aggravating factors, history of infection or trauma, noise exposure, medication usage, medical history, associated hearing loss or vertigo, family history of hearing loss and associated pain. A very important factor is to enquire about the impact of the tinnitus on the patient (Quinn \& Ryan, 2003). After a thorough history is taken, a complete head and neck examination and general physical examination should be performed by a relevant 
physician. Furthermore, patients should also undergo audiometric testing that includes pure tone audiometry, speech discrimination, tympanometry, and acoustic reflex measurement (Quinn \& Ryan, 2003).

The management of patients with tinnitus seems to be a complex challenge as tinnitus has many etiologies and its manifestation may vary from patient to patient (Vielsmeier et al., 2011). There are various claims concerning the positive effects of a variety of treatments (Henry et al., 2005); however, the area of tinnitus is fraught with controversy and conflicting information. In the past, many methods have been tried for tinnitus with variable outcomes (Henry et al., 2005). A survey of the literature indicates that the following modalities of treatment are currently being used: medical and surgical approaches, psychological approaches, Tinnitus Retraining Therapy (TRT), masking techniques, sound therapy, herbal medications and hyperbaric oxygen therapy. The two most preferred management regimes are TRT and a multidisciplinary approach (Ruth \& Hamil-Ruth, 2001).

The goal of TRT is to 'induce and facilitate the habitation of tinnitus-induced reactions and tinnitus perception resulting in a decreased awareness of tinnitus' (Bartnik, Fabijanska \& Rogowski, 2001 p. 206). Habituation to negative reactions is achieved by directive counselling and the habituation of perception of tinnitus is achieved by means of sound therapy using various acoustic backgrounds, wideband noise generators or hearing aids (Bartnik et al., 2001).

With the multidisciplinary approach, the audiologist should be the point of entry, the team leader and the coordinator in the management of tinnitus (Ruth \& Hamil-Ruth, 2001). In this setting, most patients are initially seen by the audiologist, who then makes referrals to other members deemed appropriate, but not limited to ENT specialist, medical practitioner, psychologists, psychiatrists, nurses and family members. For patients presenting with less complex problems, counselling and education by the audiologist are key elements in the management and in helping the patient understand tinnitus (Hall \& Ruth, 1999). The ENT carries out the medical evaluation and surgical management of tinnitus, if indicated. If the patient presents with pain, a physician evaluates and manages the patient for the head and neck complaints, which may compound the patient's distress. In addition, there's a need to pay attention to other treatable comorbities such as sleep disorder, depression and the presence of ototoxic medications which may further contribute to the severity of tinnitus. A referral to a psychologist to evaluate the patient's emotional, cognitive and behavioural management is also recommended. Furthermore, particular effort should be directed towards identification of any barriers to successful treatment, such as mood disorders and deficient coping strategies (Ruth \& Hamil-Ruth, 2001).

In both these treatment methods, studies indicate that audiologists are the most inherently qualified health care professionals to be the primary point for tinnitus patients (Henry et al., 2005). According to Henry et al. (2005), theoretically the role of the audiologist is well defined in the treatment of tinnitus, but what audiologists are practically doing pertaining to tinnitus treatment still needs to be determined. In addition, Henry, Zaugg, Myers, Kendall \& Michaelides (2010) argue that audiologists are knowledgeable about auditory disorders and auditory system functioning and understand acoustics and psychoacoustic, which are important components of any forms of tinnitus assessment and therapy. Furthermore, audiologists are skilled in using clinical audiometers which assess the hearing function, and they perform psychoacoustic assessment. They are also experts in the use of hearing aids, which have long been used to ameliorate the effects of tinnitus through the use of auditory stimulation. Generally, audiologists have working relationships with ENTs, who are needed for the medical evaluation and management of tinnitus (Wackym \& Friedland, 2004). And, finally, audiologists generally possess good counselling skills, which are essential for any form of tinnitus management (Hall \& Ruth, 1999).

In South Africa, there is a paucity of studies in the area of tinnitus. The literature reviewed reveals some focus on the professional clinical management of tinnitus and the nature of tinnitus in patients who present with temporomandibular disorder. Rau (2004), for example, documented the practices adopted by audiologists in private practice for treating tinnitus. This study highlighted the fact that audiologists did not feel adequately trained, nor were they adequately familiar with most techniques used to treat tinnitus. The audiologists stated that they were aware of the team approach in the treatment of tinnitus and are working with professionals including ENT specialists, psychologists, neurologists and general practitioners whilst managing the tinnitus sufferer. The main treatment methods used by audiologists in private practice in this study included counselling and referral to an ENT specialist for medical management. Another study conducted by Kanji and KhozaShangase (2013) focused on the clinical signs and symptoms in patients presenting with temporomandibular disorders (TMD). In this study, 10 patients presented with TMD and tinnitus and another group of 10 participants had tinnitus and not TMD. The findings of this study revealed clinically relevant differences in the attributes of tinnitus in patients with and without TMD. Furthermore, this study revealed a need for audiologists to play a crucial role in the correct diagnosis and treatment of tinnitus in the TMD population, and the subsequent improvement in quality of life (Kanji \& Khoza-Shangase, 2013). These two studies highlight the need for a uniform way of managing patients with tinnitus and the need for audiologists to be active in the assessment and management of patients with tinnitus regardless of its nature or cause.

It is clear from the above studies that there is a need for audiologists to be adequately trained in the assessment and management of patients with tinnitus, because studies have shown that in most patients with tinnitus, the quality of life of the patients is affected by the presence of tinnitus. International studies have alluded to the fact that depending 
on the severity of tinnitus in patients, some activities of daily living such as reading, sleeping, concentration and performing occupational tasks may be affected in patients with tinnitus.

Therefore, the aim of the current study was to describe the perceived effects of tinnitus on daily activities such as occupational performance in adults suffering from tinnitus. Perhaps the perceived effects of tinnitus on the quality of life of these patients may further emphasise the need for adequate training for audiologists and for the proper assessment and management of tinnitus in these patients. It may also highlight the need for a multidisciplinary approach to the management of tinnitus.

\section{Method \\ Objectives}

The current study aimed at describing the perceived effects of tinnitus on occupational performance and the quality of life of the participants as measured by the THI. The following were the specific sub-aims:

- to determine how tinnitus sufferers classify tinnitus as a disability

- to establish whether gender and/or age influences the classification

- To report on the coping strategies used by participants in the current study.

\section{Participants and inclusion criteria}

Participants for the current study were recruited by means of a purposive sampling strategy. This type of non-probability sampling was convenient for recruiting participants who met the inclusion criteria. The participants were recruited from public and private ENT clinics in Johannesburg; a total of 27, comprising 16 males and 11 females with an age range of 25-58 years, were included in this study. Participants had to present with tinnitus of any etiology, with or without hearing loss or other complications such as vertigo or pain or itchiness. The type of tinnitus was no barrier to the study as it focused on the presence of tinnitus. Participants had to be in employment in order to participate in this study. (See Table 1-A1 for the profile of the participants.)

\section{Research design}

This study was both qualitative and quantitative in nature since data was collected using the THI questionnaire and semi-structured interviews. A cross-sectional descriptive study design was adopted for the current study.

\section{Materials and procedures}

Data was collected from 27 participants who met the criteria for this study. Permission to conduct and collect data from different hospitals was obtained from relevant doctors in hospitals. Information letters, the consent letters and the THI questionnaires were distributed to the different hospitals and doctors were asked to pass these on to any consulting patients who presented with tinnitus. It was explained to the doctors that participation was voluntary. The doctors were requested to briefly tell their patients about the study and if the participants were willing to participate in the study, doctors were required to ask the participants to complete the consent form and the THI questionnaire. Participants were further requested to leave their contact details so that the researchers could contact them for the interviews. After a period of six weeks, the consent forms and THI questionnaires were collected from the various hospitals and the participants who left their contact details were contacted telephonically to participate in the interview. Participants who completed the THI but did not leave their contact details were not included in the study. Most participants were interviewed when they returned for further consultations or follow-ups. This was a cost-effective method as it did not seem to put a financial burden on the participants.

The THI scale was used to collect data for this study. The THI questionnaire is a disease-specific instrument which is used to measure patients' views on their tinnitus-related problems. The THI questionnaire was developed by Newman, Sandridge and Jacobson in 1998; and has been standardised on an outpatient population suffering from tinnitus. It consists of 25 questions grouped into three categories; namely: Functional disability, Emotional disability and Catastrophic disability; with a total score of 100.

For validity purposes, the THI questionnaire was administered by mail to 100 participants who presented with tinnitus, prior to undergoing any tinnitus therapy at the Cambridge Tinnitus Clinic. The response rate was 78\% and the results yielded a high convergent validity as all subscale scores significantly correlated at the 5\% level (Spearman correlation coefficients) with a high internal consistency (Cronbach's alpha of 0.93) (Bagulay, Humphriss \& Hodgson, 2000).

Participants were required to read the questions and then choose the answer that was most applicable to their condition. Each question had three answer options to choose from: 'yes', 'no' and 'sometimes' (Table 1-A2).

Following completion of the questionnaires, the participants were invited to participate in semi-structured, one-on-one interviews with the researcher. These aimed at gaining an indepth understanding of the perceived effects that tinnitus was having on their quality of life and occupational performance. On average, the interviews lasted for about 30 minutes. They were all conducted in English as all the participants were fluent in English.

The interview questions were open ended and focused on the nature and duration of the tinnitus, whether the participant was provided with and/or using any strategies to help in coping with tinnitus, whether the participants had made any environmental changes to accommodate for their tinnitus, 
and to determine what aspect of their life they perceived to be most affected by tinnitus (occupational performance or quality of life).

\section{Data analysis}

Descriptive statistics were used to analyse, present and describe the data of the THI. Furthermore, inferential statistics in the form of the independent ' $t$ ' test was utilised to establish statistically whether there was an association between gender and age and the type of perceived disability. Table 1 depicts how the scoring was conducted for the THI; it also provides a description of the various disability grading terms.

\section{Reliability and validity}

Test-retest reliability of the THI questionnaire has been shown to be high (Newman, Jacobson \& Spitzer, 1996). Validation of the THI questionnaire indicates a high internal consistency (Cronbach's alpha of 0.93) (Bagulay et al., 2000). For this study, reliability was addressed by including an independent objective peer to review and ensure the validity of the analysed data, including the data derived from the interviews.

\section{Ethical considerations}

Prior to the study being conducted, permission was obtained from the University of the Witwatersrand's Human Research Ethics Committee (Medical) (Protocol Number M070311) as well as from all other relevant authorities such as heads of departments where the data was collected. Guided by the ethical principles of the South African Medical Research Council (1993), participants were enrolled in the study following informed consent, observing confidentiality, and assuring them of their right to withdraw from the study at any point.

\section{Results}

\section{Classification of tinnitus and the influence of gender and age}

\section{Range of perception of perceived disability}

Participants presented with a wide range of perceived disability according to the THI, as depicted in Table 2. The disability rating on the THI ranged from slight to catastrophic. A surprisingly high number of participants scored on the mild range of disability.
TABLE 2: Percentage of overall disability and the classification according to the Tinnitus Handicap Inventory $(N=27)$.

\begin{tabular}{lll}
\hline Number of participants & $\mathbf{\%}$ & Classification \\
\hline 4 & 15 & Slight \\
11 & 41 & Mild \\
5 & 18 & Moderate \\
4 & 15 & Severe \\
3 & 11 & Catastrophic \\
\hline
\end{tabular}

TABLE 3: Gender breakdown of percentage of overall disability and the classification according to the Tinnitus Handicap Inventory $(N=27)$.

\begin{tabular}{llllll}
\hline $\begin{array}{l}\text { Number of } \\
\text { participants }\end{array}$ & Male $(\boldsymbol{n}=\mathbf{1 6})$ & $\mathbf{\%}$ & Female $(\boldsymbol{n}=\mathbf{1 1}) \boldsymbol{\%}$ & Classification \\
\hline 4 & 3 & 19 & 1 & 9 & Slight \\
11 & 7 & 44 & 4 & 36 & Mild \\
5 & 3 & 19 & 2 & 18 & Moderate \\
4 & 1 & 6 & 3 & 27 & Severe \\
3 & 2 & 13 & 1 & 9 & Catastrophic \\
\hline
\end{tabular}

Scores and means obtained from the tinnitus handicap inventory. $n$, number.

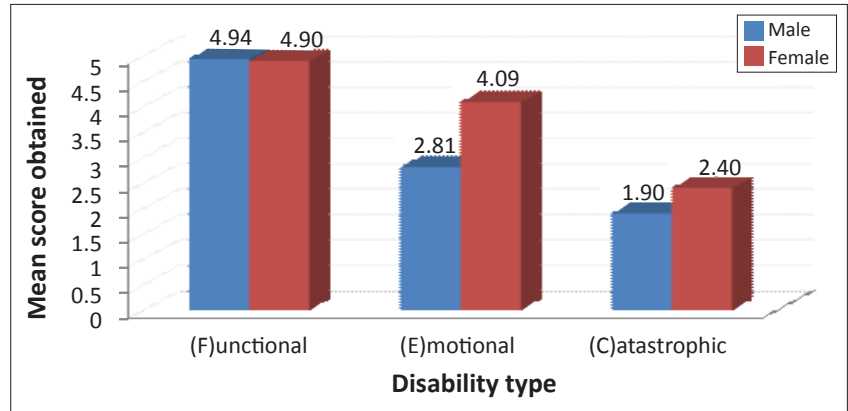

FIGURE 1: Gender comparison of functional, emotional and catastrophic disability in terms of prominence.

Nuanced analysis of the range of perception of disability according to gender yielded noteworthy results. Females tended to perceive tinnitus as more disabling than males in the current sample with more females (54\%) than males $(38 \%)$ scoring at the level of moderate to catastrophic, as depicted in Table 3.

The scores for emotional, functional and catastrophic disability were analysed separately, and these are depicted in Table 4. Scores for perceived functional disability ranged from 1 to 12 with a mean of 4.93. Perceived emotional disability ranged from 0 to 8 , with a mean of 3.33; scores for catastrophic disability ranged from 0 to 5 , with a mean of 2.11. These findings reveal that the most perceived disability on the THI seemed to be functional disability, with the least perceived disability being catastrophic for the total sample.

TABLE 1: Scoring the Tinnitus Handicap Inventory.

\begin{tabular}{llll}
\hline Scores & Description & Grade & Interpretation \\
\hline $\begin{array}{l}\text { 0-16 } \\
\text { 18-36 }\end{array}$ & $\begin{array}{l}\text { Slight disability } \\
\text { Mild disability }\end{array}$ & 1 & $\begin{array}{l}\text { Only heard in a quiet environment. Very easily masked. No interference with sleep or daily activities. } \\
\text { Easily masked by environmental sounds and easily forgotten with activities. May occasionally interfere with sleep, but not daily } \\
\text { activities. }\end{array}$ \\
36-56 & Moderate disability & 3 & $\begin{array}{l}\text { May be noticed, even in the presence of background or environmental noise, although daily activities may still be performed. } \\
\text { Less noticeable when concentrating. Not infrequently interferes with sleep and quiet activities. }\end{array}$ \\
58-76 & Severe disability & 4 & $\begin{array}{l}\text { Almost always heard; rarely, if ever, masked. Leads to disturbed sleeping patterns and can interfere with ability to carry out } \\
\text { normal daily activities. Quiet activities affected adversely. }\end{array}$ \\
\hline
\end{tabular}

Source: McCombe, A., Bagueley, D., Coles, R., McKenna, L., McKinney, C., \& Windle-Taylor, P. (2001). Guidelines for the grading of tinnitus severity: The results of a working group commissioned by the British Association of Otolaryngologists, Head and Neck Surgeons, 1999. Clinical Otolaryngol, 26, 388-393 
TABLE 4: Lowest and highest scores obtained for emotional, functional and catastrophic disability on the Tinnitus Handicap Inventory ( $N=27)$.

\begin{tabular}{|c|c|c|c|c|}
\hline Disability & Lowest score & Highest score & Mean & Total score of disability \\
\hline Functional & 1 & 12 & 4.926 & 44 \\
\hline Emotional & 0 & 8 & 3.333 & 36 \\
\hline Catastrophic & 0 & 5 & 2.111 & 20 \\
\hline
\end{tabular}

TABLE 5: The overall rating obtained by participants on the interview with regards to effects on occupational performance and quality of life: $(N=27)$.

Affected aspect of participant's life Number of participants $\quad \%$

Occupational Performance (OC) 4 - 15

Quality of Life (QoL)

Both (OC and QoL)

Neither

5

11

11

Coping strategies utilised by participants.

When nuanced analysis according to gender was done, scores for emotional, functional and catastrophic disability also revealed noteworthy findings (Figure 1). Male participants obtained a mean of 4.94 and females obtained a mean of 4.90 for perceived functional disability. For emotional disability, males obtained a mean of 2.81 and females a mean of 4.09 . For catastrophic disability, males obtained a mean of 1.90 and females 2.40. These latter two findings again highlight higher scores for females than males, yet again indicating some gender-influenced response to tinnitus.

When combining both groups (male and female), as depicted in Table 4, it was clear that for the entire group, tinnitus was perceived to cause more functional than catastrophic disability, but more nuanced analysis according to gender in Table 3 indicates different results for females.

An independent ' $t$ ' test was utilised to statistically establish if there was an association between gender and the type of perceived disability; and the findings revealed a statistically non-significant association $(p>0.05)$.

Specifically, $p$-values for functional disability were $0.34,0.44$ for emotional disability and 0.06 for catastrophic disability. These values indicate that there is no statistically significant association between the type of perceived disability and gender. This could be due to the different sample sizes for the two genders; but it does highlight the importance of multilevel analysis of data, including gender-specific analysis.

\section{Association between age and type of disability}

An independent ' $t$ ' test was used to determine whether there was an association between age and the type of perceived disability. Findings revealed that there was no statistically significant relationship between these two variables $(p>$ $0.05)$; with $p$-values being 0.08 for functional disability and 0.57 for catastrophic disability.

\section{Impact of tinnitus on occupational performance Tinnitus and its impact on occupational performance}

Table 5 indicates the significant effects of tinnitus, with a large number of the participants (41\%) reporting experiencing the effects of tinnitus at both work and on their general quality of life. A small number $(26 \%)$ reported a lack of negative effects.
Of the total sample, 18 (67\%) participants reported that they have not made any environmental or lifestyle changes to accommodate their tinnitus and $9(33 \%)$ reported to have implemented their own behavioural strategies to help cope with tinnitus. On the matter of coping strategies, 21 (78\%) participants reported that they had not been provided with any specific strategies or means to assist with the management of tinnitus. Two participants had been given hearing aids for the management of tinnitus; neither of them was still using their hearing aid, because they were not deriving any benefit from wearing them in so far as the alleviation of tinnitus was concerned.

\section{Discussion}

Participants in this study reported a wide range of perceived disability on the THI. Scores obtained by both males and females on the THI revealed overall ratings ranging from mild to catastrophic disability. This has implications for audiologists as it highlights the importance of probing further for tinnitus during the detailed case history to determine the extent to which tinnitus is affecting the individual (Newman et al., 2011; Henry, Zaugg, Myers, Kendall \& Michaelides, 2010). This will have further implications for emotional and psychosocial management arrived at ensuring the holistic management of the patient. This kind of information can be gleaned only from a case history, but, alternatively, it can also be fully obtained from routine use of tinnitus handicap scales such as the THI with all patients presenting with chronic tinnitus (Lim, Lu \& Eng, 2010). These scales would be useful not only for diagnosis but also for future comparative clinical management following tinnitus management implementation, because they would serve as baseline measures. Tools such as the THI would be useful clinical tools for audiologists, because over and above acting as part of the diagnosis and management battery, they could also provide assurance to the patient, provide the patient with a tool to demonstrate the effect of tinnitus to family members, allow for any changes in tinnitus impact over time to be maintained; and also allow for the efficacy of treatment to be determined (Tyler, 2000). This should be done bearing in mind the impact that the diversity in languages and culture can have on the understanding, acceptance and management of disease and disability.

Current findings suggest that participants experience restrictions in performing activities of daily living such as reading, concentrating on tasks, doing daily chores and during social interactions. These findings are consistent with those of Nondhal et al. (2007), where patients were extremely bothered by their tinnitus and reported that it interfered with activities of daily living. Although the current study was conducted within a developing country context; with significant socio-economic restrictions that dictate which 
health conditions take priority over others, current findings argue for the importance of hearing the voice of the sufferer in determining priorities.

Descriptively, there was a reasonably strong association between gender and type of perceived disability, although this association was found to be statistically non-significant. For example, the findings of the current study indicated that functional disability was most prominent in the whole group but more nuanced gender-based analysis revealed more effect in females. In the current sample, females tended to have more emotional and catastrophic disability than males. The current descriptive findings concur with previous studies which have suggested that men are likely to report functional disability as most prominent whilst females report emotional disability as most prominent (Lim et al., 2010). Although both males and females scored high on functional disability, the mean score for emotional disability revealed a higher score for females than for males. This suggests that females may benefit more from functional and emotional coping strategies whereas males may find it beneficial to learn functional strategies to cope with the tinnitus. The current findings also highlight the importance of counselling, which may be required for most individuals with tinnitus. The fact that there was no statistically significant association between age and perceived disability further illustrates the importance of counselling for all individuals suffering from tinnitus, regardless of age.

Although all participants in the current sample had visited the ENT specialist clinics for their initial consultation, more than half of them had not been provided with tinnitus management or coping strategies. Those who had been provided with some strategies, including amplification, did not understand the rationale or the benefits of these in tinnitus management. The provision of appropriate tinnitus management therapy to tinnitus sufferers could very well be influenced by the availability of resources, which in the South African context are sparse. Conditions that are not easily viewed as life-threatening, visibly debilitating and/ or malignant tend to receive less attention as far as resource prioritisation is concerned. The current study raises a flag about the 'invisible' significant impact of tinnitus on occupational performance and quality of life, with clear indications of how disabling the tinnitus sufferers perceive tinnitus to be. The current findings therefore highlight the importance of ensuring that tinnitus receives as much attention as any other health ailment and does not remain minimised, because the consequences of that could be far reaching; as has been previously documented.

The general practitioner, who is often the first point of contact for the patient with tinnitus, has an obligation to ensure that appropriate referrals are made to audiologists and ENT specialists (Newman et al., 2011), who would then ensure proper assessment and management of the tinnitus sufferer. It is only within a team approach that tinnitus will be appropriately and efficaciously managed as various approaches can be adopted and these require multidisciplinary collaboration, with the involvement of counsellors and/or psychologists as part of the team.

\section{Conclusion}

Although derived from a small sample size, the findings from the current pilot study highlight the fact that tinnitus may have an important and serious impact on the quality of life, daily activities and occupational performance in people suffering from it; and that this effect is perceived to be more debilitating in females than in males. This raises the need for improved awareness and understanding of the relationship between tinnitus and quality of life in general; and it also emphasises the importance of appropriate assessment and management of the tinnitus sufferer to ensure a more holistic management approach that improves the quality of life of the sufferer.

\section{Implications of the study}

The current study attempts to demonstrate the importance of having audiologists conduct appropriate assessment and management of patients presenting with tinnitus to ensure a positive quality of life. This is because audiologists can play a significant role in the correct diagnosis and treatment of tinnitus, subsequently improving the quality of life in individuals suffering from tinnitus regardless of the etiology or the onset. This study also highlights the need for a uniform approach to treating tinnitus to be developed and for audiologists to be adequately trained in handling tinnitus patients. It also raises questions about the need for a multidisciplinary approach to tinnitus management. This study further advocates the use of the THI questionnaire for the assessment and management of tinnitus as the THI can alert the therapist to the impact the tinnitus has on the individual based on the score obtained. Furthermore, the THI questionnaire may help to identify patients who may need referrals to other professionals such as psychologists and counsellors for further management.

The current findings should be viewed within the identified limitations of the design and implementation of the study. These limitations include the fact that the sample size was small; it also consisted only of employed adult participants in one region of the country, with an unequal gender distribution. This therefore limits the generalisability of the findings to the general tinnitus-suffering population. But, nonetheless, it provides enough preliminary evidence to highlight the importance of further studies within this context, looking at the suffering individual within the ICF model of care.

Further research should be conducted to determine the effects of tinnitus on specific trades and professions such as those in mines, construction sites and call centres. Such research should also seek to determine the effects of socioeconomic status, levels of education and personality traits of individuals on the perceived disability, all of this performed on a larger sample size to overcome the statistical analysis limitations identified in the current study. 


\section{Acknowledgements Competing interests}

The authors declare that they have no financial or personal relationship(s) that may have inappropriately influenced them in writing this article.

\section{Authors' contributions}

N.F.M. (University of Witwatersrand) was the project leader and the chief researcher for this project. K.K.S. (University of the Witwatersrand) was responsible for the analysis of the data for this project.

\section{References}

Bagulay, D.M., Humphriss, R.L., \& Hodgson, C.A. (2000). Convergent validity of the tinnitus handicap inventory and the tinnitus questionnaire. Journal of Laryngology and Otology, 114(11), 840-843.

Bartnik, G., Fabijanska, A., \& Rogowski, M. (2001). Effects of tinnitus retraining therapy (TRT) for patients with tinnitus and subjective hearing loss versus tinnitus only. Scandinavian Audiology, 30(52), 206-208. http://dx.doi. org/10.1080/010503901300007542

Baldo, P., Doree, C., Lazzarini, R., Molin, P., \& McFerran, D. (2006). Antidepressants for patients with tinnitus. Cochrane Database of Systematic Reviews, 4, 1-27.

Davies, A., \& Refaie, A. (2000). Epidemiology of tinnitus. In R.S. Tyler (Ed.), Tinnitus handbook, (pp. 25-58), San Diego, CA: Singular Publishing Group.

Dineen, R., Doyle, J., \& Bench, J. (1997). Audiological and psychological charactersitics of a group of tinnitus sufferers, prior to tinnitus management training. British Journal of Audiology, 31, 27-38. http://dx.doi.org/10.3109/03005364000000006

Erlandsson, S.I., Hallberg, L.R-M., \& Axelsson, A. (1992). Psychological and audiological correlates of perceived tinnitus severity. Audiology, 31, 168-179. http://dx.doi. org/10.3109/00206099209072912

Erlandsson, S.I., \& Holgers, K-M. (2001). The impact of perceived tinnitus severity on health-related quality of life with aspects of gender. Noise \& Health, 3(10), 39-51.

Gopinath, B., McMahon, C.M., Rochtchina, E., Karpa, M.J., \& Mitchell, P. (2010). Risk factors and impacts of incidental tinnitus in older adults. Annals of Epidemiology, 20(2), 129-135. http://dx.doi.org/10.1016/j.annepidem.2009.09.002

Hall, J.W., III, \& Ruth, R.A. (1999). Outcome for tinnitus patients after consultation with an audiologist. In J. Hazell (Ed.), Sixth international Tinnitus seminar (pp. 378-380). London: The Tinnitus and Hyperacusis Centre.

Henry, J.A., Dennis, K.C., \& Schechter, M.A. (2005). General review of tinnitus: Prevalence, mechanisms, effects and management. Journal of Speech, Language and Hearing Research, 48(5), 1204-1232. http://dx.doi.org/10.1044/1092 4388(2005/084)

Henry, J.A., Zaugg, T.L., Myers, P.J., Kendall, C.J., \& Michaelides, E.M. (2010). A triage guide for tinnitus: Ringing in the ears may be symptomatic of a serious condition or it may be benign. This guide can help you tell the difference. Journal of Family or it may be benign. This
Practice, 59(7), 389-393.

Hiller, W. \& Goebel, G. (2007). When tinnitus loudness and annoyance are discrepant: Audiological characteristics and psychological profile. Audiology and NeuroOtology, 27(12), 391-400. http://dx.doi.org/10.1159/000106482

Jacobson, G.P., \& McCaslin, D.L. (2001). A search for evidence of a direct relationship between tinnitus and suicide. Journal of American Academy of Audiology, 12, 493-496.

Jastreboff, P.J., \& Hazell, J.W.P. (1993). A neurophysiological approach to tinnitus: Clinical implications. British Journal of Audiology, 27, 7-17. http://dx.doi. org/10.3109/03005369309077884

Kanji, A., \& Khoza-Shangase, K. (2013). Clinical signs and symptoms of tinnitus in temporomandibular joint disorders: A pilot study comparing patients and nonpatients. South African Journal of Communication Disorders, 60(1), 16-20. http:// dx.doi.org/10.7196/sajcd.248

Krog, N.H., Engdahl, B., \& Tambs, K. (2010). The association between tinnitus and mental health in a general population sample: Results from the HUN study. Journal of Psychosomatic, 69, 289-298. http://dx.doi.org/10.1016/j. jpsychores.2010.03.008
Lewis, J.E., Stephens, S.D., \& Mckenna, L. (1994). Tinnitus and suicide Clinical Otolaryngology of Allied Science, 19(5), 50-54. http://dx.doi. org/10.1111/j.1365-2273.1994.tb01147.x

Lim, J.J.B.H., Lu, P.K.S., \& Eng, S.P. (2010). The impact of tinnitus as measured by the Tinnitus Handicap Inventory among tinnitus sufferers in Singapore. Singapore Medical Journal, 51(7), 551

Martines, F., Bentivegna, D., Martines, E., Sciacca, V., \& Martinciglio, G. (2010) Characteristics of tinnitus with or without hearing loss: Clinical observations in Sicilian tinnitus patients. Auris Nasus Larynx: International Journal of ORL \& HNS, 37, 685-693. http://dx.doi.org/10.1016/j.anl.2010.03.008

McCombe, A., Bagueley, D., Coles, R., McKenna, L., McKinney, C., \& Windle-Taylor P. (2001). Guidelines for the grading of tinnitus severity: the results of a workin group commissioned by the British Association of Otolaryngologists, Head and Neck Surgeons, 1999. Clinical Otolaryngology, 26, 388-393. http://dx.doi. org/10.1046/j.1365-2273.2001.00490.x

McDougall, J., Horgan, K., Baldwin P., Tucker M.A., \& Frid, P. (2008). Employing the international classification of functioning, disability and health to enhance services for children and youth with chronic physical health conditions and disabilities. Paediatric Child Health, 13(3), 173-178.

Meikle, M.B. (2003). A conceptual framework to aid the diagnosis and treatment of severe tinnitus. The Australian and New Zealand Journal of Audiology, 24, 59-67. http://dx.doi.org/10.1375/audi.24.2.59.31107

Newman, C., Jacobson, G.P., \& Spitzer, J.B. (1996). Development of the Tinnitus Handicap Inventory. Archives of Otolaryngology: Head Neck Surgery, 122, 143148. http://dx.doi.org/10.1001/archotol.1996.01890140029007

Newman, C.W., Sandridge, S.A., Bea, S.M., Cherian, K., Cherian, N., Kahn, K.M., \& Kaltenbach, J. (2011). Tinnitus: patients do not have to 'just live with it'. Cleveland
Clinical Journal of Medicine, 78(5), 312-319. http://dx.doi.org/10.3949/ Clinical Journal

Newman, C.W., Sandridge, S.A., \& Jacobson, G. (1998). Psychometric adequacy of the tinnitus handicap inventory for evaluating treatment outcome. Journal of the tinnitus handicap inventory for evaluati
American Academy of Audiology, 9, 153-160.

Nondahl, D.M., Cruickshanks, K.J., Dalton, D.S., Klein, B.E.K., Klein, R., Schubert, C.R., \& Wiley, T.L. (2007). The impact of tinnitus on quality of life in older adults. Journal of the American Acaddamy of Audiology, 18, 257-266. http://dx.doi.org/10.3766/ jaaa.18.3.7

Pinto, P.C.L., Sanchez, T.G., \& Tomita, S. (2010). The impact of gender, age and hearing loss on tinnitus severity. Brazilian Journal of Otorhinolaryngology, 76(1), 18-24.

Quinn, F.B., \& Ryan, M.W. (2003, January). Tinnitus. Grand Rounds Presentation, Department of Otolaryngology, University of Texas Medical Branch, Galveston TX.

Ramkumar, V., \& Rangasayee, R. (2010). Studying tinnitus in the ICF framework. International Journal of Audiology, 49, 645-650. http://dx.doi.org/10.3109/149 92027.2010.484828

Rau, E. (2004). Management of subjective tinnitus by audiologist in private practice in Gauteng. Undergraduate study submitted to the University of Witwatersrand, Johannesburg, South Africa.

Roggerone, M. (2010). Determinants of tinnitus' impact in quality of life in an outpatient clinic protocol. Groningen: University Medical Centre.

Ruth, R.A., \& Hamil-Ruth, R. (2001). A multidisciplinary approach to the management of tinnitus and hyperacusis. Hearing Journal, 54, 26-32. http://dx.doi. org/10.1097/01.HJ.0000293151.70972.56

South African Medical Research Council. (1993). Guidelines on ethics for medical research. Retrieved March 21, 2012, from http://www.sahealthinfo.org/ethics/ ethicsreferences.htm

Stevinson, E.E. (2003). Ginkgo biloba may help tinnitus, but needs further investigation British Medical Journal, 327(7416), 630

Stobik, C., Weber, R.K., Münte, T.F., Walter, M., \& Frommer, J. (2005). Evidence of psychosomatic influences in compensated and decompensated tinnitus. International Journal of Audiology, 44(6), 370-378. http://dx.doi. org/10.1080/14992020500147557

Tyler, R.S. (2000). Tinnitus handbook. San Diego, CA: Singular Publication Group.

Vielsmeier, V., Kleinjung, T., Strutz, J., Burgers, R., Kreuzer, P.M., \& Langguth, B. (2011). Tinnitus with temporomandibular joint disorders: A specific entity of tinnitus
patients. Otolaryngology - Head and Neck Surgery, 145(5), 748-752. http:// patients. Otolaryngology - Head and Nec
dx.doi.org/10.1177/0194599811413376

Wackym, P.A., \& Friedland, D.R. (2004). Otologic evaluation. In J.B. Snow (Ed.), Tinnitus: Theory and management (pp. 1-7). Lewiston, NY: BC Decker Inc.

Westin, V., Hayes, S.C., \& Andersson, G. (2008). Is it the sound or my relationship to it? The role of acceptance in predicting tinnitus impact. Behaviour Research and Therapy, 46, 1259-1265. http://dx.doi.org/10.1016/j.brat.2008.08.008 


\section{Appendix 1}

TABLE 1-A1: Profile of the participants.

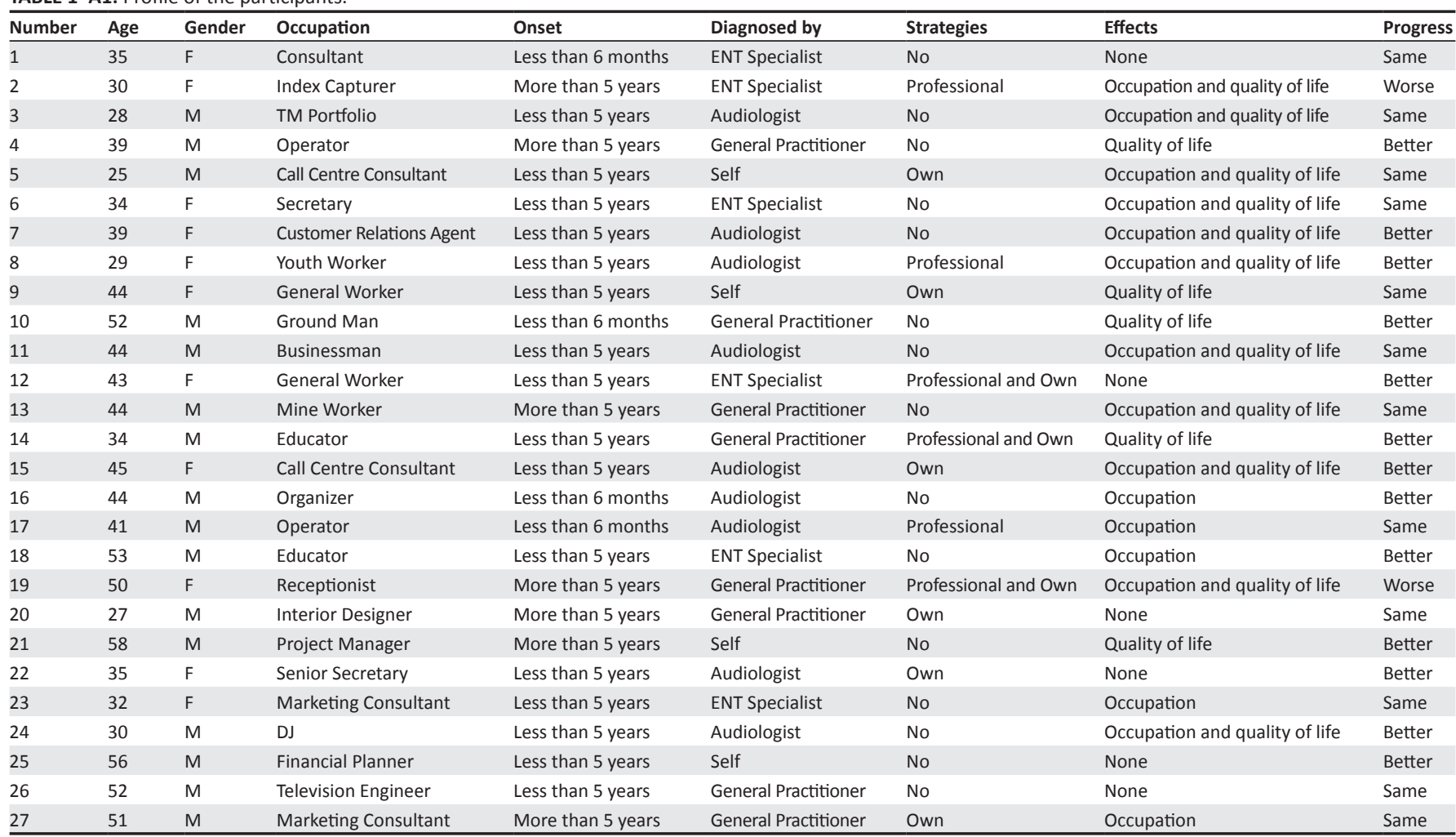

F, female; M, male; TM, Trademark; DJ, Disc Jockey; ENT, ear, nose and throat. 


\section{Appendix 2}

TABLE 1-A2: Tinnitus Handicap Inventory.

\begin{tabular}{|c|c|c|c|c|}
\hline \multirow[t]{2}{*}{ Number } & \multirow[t]{2}{*}{ Question } & \multicolumn{3}{|c|}{ Points } \\
\hline & & 4 & 0 & 2 \\
\hline 1 & Because of your Tinnitus is it difficult for you to concentrate? & Yes & No & Sometimes \\
\hline 2 & Does the loudness of your Tinnitus make it difficult for you to hear people? & Yes & No & Sometimes \\
\hline 4 & Does your Tinnitus make you confused? & Yes & No & Sometimes \\
\hline 5 & Because of your Tinnitus are you desperate? & Yes & No & Sometimes \\
\hline 6 & Do you complain a great deal about your Tinnitus? & Yes & No & Sometimes \\
\hline 8 & Do you feel as though you cannot escape from your Tinnitus? & Yes & No & Sometimes \\
\hline 9 & Does your Tinnitus interfere with your ability to enjoy social activities (such as going out to dinner, to the cinema)? & Yes & No & Sometimes \\
\hline 10 & Because of your Tinnitus do you feel frustrated? & Yes & No & Sometimes \\
\hline 11 & Because of your Tinnitus do you feel that you have a terrible disease? & Yes & No & Sometimes \\
\hline 12 & Does your Tinnitus make it difficult to enjoy life? & Yes & No & Sometimes \\
\hline 13 & Does your Tinnitus interfere with your job or household responsibilities? & Yes & No & Sometimes \\
\hline 14 & Because of your Tinnitus do you find that you are often irritable? & Yes & No & Sometimes \\
\hline 16 & Does your Tinnitus make you upset? & Yes & No & Sometimes \\
\hline 17 & Do you feel that your Tinnitus has placed stress on your relationships with members of your family and friends? & Yes & No & Sometimes \\
\hline 18 & Do you find it difficult to focus your attention away from your Tinnitus and on to other things? & Yes & No & Sometimes \\
\hline 19 & Do you feel that you have no control over your Tinnitus? & Yes & No & Sometimes \\
\hline 20 & Because of your Tinnitus do you often feel tired? & Yes & No & Sometimes \\
\hline 21 & Because of your Tinnitus do you feel depressed? & Yes & No & Sometimes \\
\hline 22 & Does your Tinnitus make you feel anxious? & Yes & No & Sometimes \\
\hline 23 & Do you feel you can no longer cope with your Tinnitus? & Yes & No & Sometimes \\
\hline 24 & Does your Tinnitus get worse when you are under stress? & Yes & No & Sometimes \\
\hline 25 & Does your Tinnitus make you feel insecure? & Yes & No & Sometimes \\
\hline
\end{tabular}

Source: Newman, C., Jacobson, G.P., \& Spitzer, J.B. (1996). Development of the Tinnitus Handicap Inventory. Archives of Otolaryngology: Head Neck Surgery, 122(2), 143-148. http://dx.doi. org/10.1001/archotol.1996.01890140029007 